Pesq. Vet. Bras. 38(5):875-882, maio 2018

\title{
Claviceps purpurea e Bipolaris sp. como causa de ergotismo em bovinos no estado de Santa Catarina ${ }^{1}$
}

\author{
Deise Hemckmeier ${ }^{2 *}$, Claudia M. Galindo ${ }^{3}$, Elaine Melchioretto ${ }^{4}$, \\ Aldo Gava ${ }^{4}$ e Ricardo T. Casa ${ }^{5}$
}

\begin{abstract}
Hemckmeier D., Galindo C.M., Melchioretto E., Gava A. \& Casa R.T. 2018. [Claviceps purpurea e Bipolaris sp. as a cause of ergotism in cattle in Santa Catarina State.] Claviceps purpurea e Bipolaris sp. como causa de ergotismo em bovinos no estado de Santa Catarina. Pesquisa Veterinária Brasileira 38(5):875-882. Laboratório de Patologia Animal, Centro de Ciências Agroveterinárias, Universidade do Estado de Santa Catarina, Avenida Luís de Camões 2090, Bairro Conta Dinheiro, Lages, SC 88520-000, Brazil. E-mail: a6.deise.hemckmeier@gmail.com

Claviceps purpurea is the fungus associated with ergotism. Ergotism is a disease caused by the ingestion of esclerodios called ergot, which contains alkaloids that act on dopaminergic and adrenergic receptors, causing seratoninergic effect on blood vessels, smooth muscles and central nervous and autonomic system. The present study describes epidemiological data, clinical signs and lesions of a bovine cattle disease characterized by hyperthermia, tachycardia, tachypnea and injuries in the coronary band of the hooves. Initially the cause was attributed to the fungus Claviceps purpurea. Between 2000 and 2014, 13 outbreaks were described, being three of distermic form, seven of gangrenous and three of the convulsive form. However, in five out of the seven of the gangrenous form outbreaks, it has been observed a high incidence of smut grass (Sporobolus indicus) inflorescences infected by a blackened fungus, classified as Bipolaris sp., in the places where the bovine grazed. The disease was reproduced experimentally by administration of inflorescences of smut grass contaminated by Bipolaris sp. Five bovine received daily doses of $0.1 \mathrm{~g} / \mathrm{kg}, 0.2 \mathrm{~g} / \mathrm{kg}, 0.2 \mathrm{~g} / \mathrm{kg}, 0.26 \mathrm{~g} / \mathrm{kg}$ and $0.34 \mathrm{~g} / \mathrm{kg}$ during $4,7,9,30$ and 30 days respectively. After three to seven days of eating contaminated inflorescences four cattle had diarrhea, tachycardia, tachypnea and intermittent hyperthermia. These clinical signs happened on the warmest days and during the warmest temperatures of the day. A bovine showed hyperemia in the coronary band of the hoof and loss of tail tip hair. According to data obtained during the experimental reproduction, smut grass contaminated by Bipolaris sp. can cause hyperthermia, tachycardia, tachypnea, injuries in the coronary band of the hoof and loss of the tail tip hair in bovine cattle.
\end{abstract}

INDEX TERMS: Claviceps purpurea, Bipolaris sp., ergotism, Sporobolus indicus, cattle, fungus, toxicoses.

\footnotetext{
${ }^{1}$ Recebido em 3 de maio de 2017.

Aceito para publicação em 10 de junho de 2017.

Dissertação de Mestrado do primeiro autor, defendida na Universidade do Estado de Santa Catarina (UDESC).

${ }^{2}$ Secretaria Municipal de Agricultura, Rua Henrique Bichels s/n, Centro, Pouso Redondo, SC 89172-000, Brasil. *Autor para correspondência: a6.deise.hemckmeier@gmail.com

${ }^{3}$ Laboratório de Patologia Animal, Hospital Veterinário, Universidade Federal do Paraná (UFPR), Rua dos Funcionários 1540, Curitiba, PR 80035-050, Brasil.

${ }^{4}$ Laboratório de Patologia Animal, Centro de Ciências Agroveterinárias (CAV), Universidade do Estado de Santa Catarina (UDESC), Av. Luiz de Camões 2090, Bairro Conta Dinheiro, Lages, SC 88520-000, Brasil.

${ }^{5}$ Laboratório de Fitopatologia, Centro de Ciências Agroveterinárias (CAV), Universidade do Estado de Santa Catarina (UDESC), Av. Luiz de Camões 2090, Bairro Conta Dinheiro, Lages, SC 88520-000, Brasil.
}

RESUMO.- Claviceps purpurea é o fungo associado ao ergotismo. Esta é uma enfermidade causada pela ingestão de escleródios chamados de ergot, que contém alcalóides que atuam em receptores adrenérgicos, dopaminérgicos e seratoninérgicos causando efeito direto em vasos sanguíneos, musculatura lisa e sistema nervoso central e autônomo. Descrevem-se dados epidemiológicos, sinais clínicos e lesões de uma enfermidade de bovinos caracterizada por hipertermia, taquicardia, taquipneia e ulcerações na coroa do casco de bovinos. Entre 2000 e 2014 foram diagnosticados 13 surtos, três da forma distérmica, sete da forma gangrenosa e três da forma convulsiva. Porém, em cinco destes sete surtos, nos locais onde esses animais pastoreavam havia alta incidência de 
inflorescências de Sporobolus indicus conhecido como "capimmourão", infectadas por um fungo com coloração enegrecida, identificado como Bipolaris sp. A enfermidade foi reproduzida experimentalmente em cinco bovinos com a administração destas inflorescências. Os bovinos em experimentação receberam inflorescências de $S$. indicus respectivamente nas doses diárias de $0,1 \mathrm{~g} / \mathrm{kg}, 0,2 \mathrm{~g} / \mathrm{kg}, 0,2 \mathrm{~g} / \mathrm{kg}, 0,26 \mathrm{~g} / \mathrm{kg}$ e 0,34g/ $\mathrm{kg}$ por um período de 4, 7, 9 e 30 dias. Após três a sete dias de ingestão das inflorescências infectadas, quatro dos cinco bovinos apresentaram diarreia e manifestaram taquicardia, taquipneia e hipertermia em algum período durante a ingestão. Estes sinais coincidiram com os dias mais quentes deste período. Um bovino manifestou hiperemia na coroa do casco e perda de pêlos da extremidade da cauda. Baseado na reprodução experimental é possível afirmar que o capim Sporobolus indicus infectado pelo fungo Bipolaris sp é capaz de causar diarreia, hipertermia, taquicardia, taquipneia, hiperemia na coroa do casco e perda de pêlos da extremidade da cauda.

TERMOS DE INDEXAÇÃO: Claviceps purpurea, Bipolaris sp., Sporobolus indicus, fungo, bovinos, toxicoses.

\section{INTRODUÇÃO}

Claviceps purpurea é descrito como um fungo Ascomycota, da ordem Hypocreales, que se caracteriza por atingir sementes de gramíneas (Alexopoulos \& Mims 1996). Centeio (Secale cereale) e outros cereais como trigo (Triticum aestivum), cevada (Hordeum vulgare), aveia (Avena sativa), (Avena strigosa) sorgum (Sorghum vulgare), arroz (Oriza sativa) (Copetti et al. 2002) e muitos capins, como Phleum pratense (capim-timóteo), Poa sp., Calamagrostis sp., Bromus sp., Dactylis sp., Paspalum sp., Agropyron sp., Agrostis sp., Phalaris sp., Phleum sp., Agropyron sp. (Coppock et al. 1989) Cynosurus cristatus, Brachiaria decumbens, Brachiaria humidicola e Pennisetum typhoides (Radostits et al. 2002). No Uruguai é observado em azevém (Lolium multiflorum), Festuca spp., Phalaris spp., aveia e trigo (Riet-Correa 1993). No Rio Grande do Sul, o fungo Claviceps purpurea tem sido observado em Lolium multiflorum, Hoicus lanatus, Setaria geniculata, Chtotropis chilensis, Poa pratensis, Festuca arudinacea, Festuca rubra e Festuca sp. (Luzzardi \& Oliveira 1973).

A ingestão de grandes quantidades de sementes infectadas com escleródios (esporões) de Claviceps purpurea causa o ergotismo em bovinos, ovinos, suínos, equinos, cães, aves (Riet-Correa 1993, Quinn et al. 2005b) e humanos (Belser-Ehrlich et al. 2013, Schneider et al. 1996). A intoxicação ocorre tanto em animais que recebem alimentação preparada a partir de grãos contaminados (Schneider et al. 1996, Ilha et al. 2001, Khalloub et al. 2007) como feno e silagem (Woods et al. 1966), quanto em animais a pasto (Appleyard 1986, Bourke et al. 2000). 0 fungo C. purpurea possui alcalóides peptídeos (Mantle 1969, Khalloub et al. 2007) divididos em dois grupos estruturais: alcalóides de aminoácidos (ergotamina) e alcalóides de aminas e compostos afins (ácido lisérgico e ergonovina).

Todos os alcalóides do ergot provocam elevação da pressão arterial, em consequência da vasoconstrição periférica, que é mais pronunciada nos vasos pós-capilares que nos pré-capilares. Em geral, estes compostos produzem ainda bradicardia, mesmo quando a pressão sanguínea está elevada, devido ao aumento da atividade vagal ou ainda depressão direta do miocárdio (Vital \& Acco 2006).
Nos bovinos o fungo C. purpurea produz 4 formas clínicas distintas: distérmica, gangrenosa, reprodutiva, e nervosa (Riet-Correa 1993). A forma distérmica é caracterizada por temperatura corporal elevada, entre $40^{\circ} \mathrm{C} \mathrm{e} 42^{\circ} \mathrm{C}$ (Ross et al. 1989, Peet et al. 1991, Rissi et al. 2007), que pode chegar a $43^{\circ} \mathrm{C}$ (Bourke et al. 2000), hipersalivação, dispneia (Jessep et al. 1987, Ilha et al. 2003), descarga nasal (Peet et al. 1991) sendo que alguns bovinos permanecem com a boca aberta e a língua fora da cavidade bucal. Além disso, podem apresentar pêlo comprido e sem brilho (Schneider et al. 1996), diminuição do consumo de alimentos, chegando a reduzir 10-50\% da ingestão (Ilha et al. 2003), aumento do consumo de água, poliúria, diminuição da produção de leite (Jessep et al. 1987), podendo reduzir em 75\% e, em casos extremos até 95-100\% (Ilha et al. 2003), antecipação do período seco, além da redução no ganho de peso (Jessep et al. 1987, Schneider et al. 1996). À noite, quando as temperaturas são mais amenas, os animais voltam a consumir alimentos quase que normalmente (Jessep et al. 1987). Muitos animais a presentam-se aparentemente normais em dias com temperatura amena, mas os sinais reaparecem em dias quentes (Schneider et al. 1996, Bourke 2003). Bovinos Bos taurus são mais severamente afetados que raças Bos indicus (Peet et al. 1991).

A forma gangrenosa é caracterizada por necrose de extremidades, resultante da vasoconstrição periférica (Appleyard 1986) e, acomete particularmente as partes inferiores dos membros posteriores (Woods et al. 1966, Schneider et al. 1996). As lesões também são observadas em cauda, orelhas e na mucosa oral (Coppock et al. 1989). Esta forma ocorre principalmente no inverno (Belser-Ehrlich et al. 2013), caracterizando-se por gangrena seca das extremidades, sendo inicialmente observados vermelhidão, tumefação, esfriamento e perda de pêlos e claudicação com progressiva perda da sensibilidade. 0 quadro evolui para o aparecimento de uma coloração azul a preta, ressecamento da pele seguida da separação dos tecidos normais (Woods et al. 1966, McKeon \& Egan 1971, Belser-Ehrlich et al. 2013).

A forma nervosa é pouco frequente em bovinos, e é caracterizada por cambaleio, sonolência (Quinn et al. 2005b), tremores, incoordenação, cegueira aparente, opistótono, convulsões, paralisia posterior e decúbito. Esta forma não foi reproduzida experimentalmente (Burfening 1973).

A forma reprodutiva é descrita com mais frequência em éguas, sendo que nesta espécie ocorrem abortos, distocia por falta de dilatação cervical, espessamento dos envoltórios fetais, retenção das membranas fetais, ruptura uterina, gestação prolongada, nascimento de potros fracos ou mortos, agalactia. Morte embrionária, anestro e esterilidade também são relatados. Quanto aos sinais clínicos dos potros nascidos, os principais são fraqueza, incapacidade de ficar em pé, ausência do reflexo mamário (Riet-Correa et al. 1988, Copetti et al. 2002). Em bovinos, já foram descritos distocia, nascimento de bezerros natimortos, retenção de placenta, agalactia (Appleyard 1986), baixa taxa de concepção, aborto e nascimento de bezerros prematuros vivos, porém com atrofias (Ilha et al. 2003). Os abortos ocorrem entre o quinto e a metade do oitavo mês e podem ser observados ainda casos de fetos mumificados e anêmicos, em consequência da vasoconstrição (Mantle \& Gunner 1965a, 1965b).

Bipolaris sp. é um fungo Deuteromycota pertencente a classe Hyphomycetes e a família Dematiacea. A reprodução 
normalmente é assexuada através de conídios, cilíndricos, escuros, grandes e multicelulares. Nos vegetais, o gênero Bipolaris causa lesões caracterizadas por manchas foliares (Alexopoulos \& Mims 1996).

Algumas espécies de Bipolaris são descritas como causadores de enfermidades em animais (Waurzyniak et al. 1992, Quinn et al. 2005a) e humanos (Gourley et al. 1990, Saubolle \& Sutton 1996, Koshy \& Daniel 2002). Bipolaris spicifera é descrito como agente causador de micose subcutânea em gatos, cães, equinos, bovinos (Quinn et al. 2005b) além de ocasionar micetoma (Fontes \& Abreu 2015), ceratomicose (Saha \& Das 2005), feohifomicose nasal em humanos (Rao et al. 1989) e lesões de pele e encéfalo secundárias a embolia fúngica em humanos imunossuprimidos (Teran et al. 2014). El Khizzi et al. (2010) descrevem o gênero Bipolaris como fungos causadores de diversas enfermidades em humanos imunodeficientes (McGinnis et al. 1986) e imunocompetentes (Rao et al. 1989). 0 gênero Bipolaris também é descrito como etiologia de feohifomicose cerebral em cães (Giri et al. 2011) e já foi isolado da conjuntiva de equino clinicamente saudáveis (Sousa et al. 2011).

Sporobolus indicus é uma gramínea nativa da América tropical e subtropical, atualmente disseminada em regiões quentes do mundo, sendo conhecido como "capim-capeta", "capim-mourão", "capim-moirão" e "capim-cortisia" (Kissmann \& Groth 1997). No Brasil está presente em quase todo o território. É classificada como planta invasora de áreas destinadas a pastagens e lavouras perenes, pode ser observada em campos nativos, áreas desocupadas, beiras de estradas, carreadores, terrenos baldios e gramados (Kissmann \& Groth 1997, Lorenzi 2008).

\section{MATERIAL E MÉTODOS}

Intoxicação espontânea. Informações sobre uma enfermidade com quadro clínico patológico compatível com ergotismo foram obtidos nos arquivos do Laboratório de Patologia Animal da Universidade do Estado de Santa Catarina (LAPA/CAV-UDESC). Sementes de aveia (Avena strigosa) e de azevém (Lolium sp.) foram coletadas de uma propriedade localizada no município de Campo Erê/SC, onde ocorreu um dos surtos da doença espontânea e foram analisadas no Laboratório de Fitopatologia do Centro de Ciências Agroveterinárias da Universidade do Estado de Santa Catarina.

Intoxicação experimental. Inflorescências da planta Sporobolus indicus infectadas por fungo foram colhidas semanalmente nos municípios de Lages e Bom Retiro. As inflorescências foram pesadas e armazenadas em sacos de papel nas doses diárias a serem fornecidas. Estas foram administradas via oral para cinco bovinos machos. Amostras destas inflorescências foram encaminhadas ao Laboratório de Fitopatologia do Centro de Ciências Agroveterinárias da Universidade do Estado de Santa Catarina para descrição do agente causal.

Os cinco bovinos utilizados no experimento foram mantidos em piquetes com capim quicuio (Pennisetum clandestinum) e água ad libitum e foram submetidos a exames clínicos diários durante o período do experimento até 30 dias após o fornecimento das inflorescências de $S$. indicus. Foram avaliados temperatura retal, frequência cardíaca e respiratória, coloração de mucosas e alterações no comportamento. Os valores considerados normais para a espécie foram para frequência cardíaca 70 e 90 batimentos por minuto, frequência respiratória entre 30 e 45 movimentos respiratórios por minuto. E valores limite para temperatura de $39,5^{\circ} \mathrm{C}$ (Stöber 1993). A temperatura corpórea diária de cada bovino foi correlacionada a temperatura ambiente, cujos dados foram obtidos através da Empresa de Pesquisa Agropecuária e Extensão Rural de Santa Catarina, por meio do Centro de Informações de Recursos Ambientais e de Hidrometeorologia de Santa Catarina - Epagri/Ciram.

O delineamento dos experimentos com inflorescências de S. indicus infectadas por fungo estão representadas no Quadro 1. 0 experimento foi realizado de acordo com a aprovação pelo Comitê de Ética em Experimentação Animal da Universidade do Estado de Santa Catarina (CETEA/UDESC), protocolo 1.05.15.

\section{RESULTADOS E DISCUSSÃO}

\section{Intoxicação espontânea}

No período de 2000 a 2014 foram acompanhados 13 surtos, de uma enfermidade compatível com ergotismo, sendo três caracterizados pela forma distérmica, sete da forma gangrenosa e três da forma nervosa (Quadro 2). Com exceção de um surto da forma distérmica em que houve isolamento laboratorial do fungo Claviceps purpurea, todos os demais foram diagnosticados com base na epidemiologia, manifestações clínicas associadas a ingestão de cereais contaminados por fungo morfologicamente semelhante ao $C$. purpurea. A forma distérmica foi observada em vacas leiteiras, da raça holandesa, alimentadas com sementes de aveia e, ou, em pastoreio direto sobre azevém, e, ou aveia em fase de maturação das sementes. Os bovinos acometidos manifestaram queda da produção leiteira, hipertermia, respiração rápida e com língua exposta, e alguns apresentaram claudicação em um ou mais membros.

Na maioria dos surtos da forma gangrenosa, os bovinos permaneciam em pastagem nativa invadida pelo capim Sporobolus indicus na fase de maturação das inflorescências, estas contaminadas por fungos. Em dois Surtos (9 e 10) os bovinos permaneciam em pastagem de Brachiaria decumbens com sementes e ração comercial como suplemento e com silagem de milho, farelo de trigo e milho em grão, respectivamente. Os principais sinais clínicos foram dificuldade de locomoção, principalmente, por locais onde o piso era cimentado, tinham

Quadro 1. Delineamento dos experimentos com inflorescências de Sporobolus indicus infectadas com fungo Bipolaris sp.

\begin{tabular}{|c|c|c|c|c|c|}
\hline Número do bovino & $\begin{array}{c}\text { Idade } \\
\text { (meses) }\end{array}$ & $\begin{array}{l}\text { Peso } \\
(\mathrm{kg})\end{array}$ & Dose diária $(\mathrm{g} / \mathrm{kg})$ & $\begin{array}{c}\text { Dias } \\
\text { de ingestão }\end{array}$ & $\begin{array}{c}\text { Dose Total } \\
\text { (gramas) }\end{array}$ \\
\hline 2 & 9 & 193 & 0,26 & 7 & 350 \\
\hline 4 & 14 & 354 & 0,1 & 30 & 1.050 \\
\hline 5 & 14 & 356 & 0,2 & 9 & 630 \\
\hline
\end{tabular}


Quadro 2. Resumo dos surtos de ergotismo registrados no Laboratório de Patologia Animal no período de 2000-2014

\begin{tabular}{cccc}
\hline Forma de intoxicação & Município & Número de animais & Alimentação \\
\hline Distérmica & Xanxerê (Surto1) & $5 / 13$ & $\begin{array}{c}\text { Pastagem Azevém e Milheto } \\
\text { Silagem de milho, ração comercial } \\
\text { Aveia em grão }\end{array}$ \\
Xanxerê (Surto 2) & $3 / 15$ & $\begin{array}{c}\text { Pastagem Azevém e Milheto } \\
\text { Silagem de milho, Ração comercial } \\
\text { Aveia em grão } \\
\text { Pastagem nativa } \\
\text { Aveia e trigo (grãos) }\end{array}$ \\
Gangrenosa & Campo Erê (Surto 3) & $34 / 70$ & Pastagem nativa invadida por Sporobolus indicus \\
& Bom Retiro (Surto 4) & $6 / 8$ & Pastagem nativa invadida por Sporobolus indicus \\
& Bom Retiro (Surto 5) & $8 / 12$ & Pastagem trevo invadida por Sporobolus indicus \\
& Lages (Surto 6) & $8 / 30$ & Pastagem trevo invadida por Sporobolus indicus \\
& Lages (Surto7) & $4 / 30$ & Pastagem nativa invadida por Sporobolus indicus \\
& Vargeão (Surto 8) & $90 / 325$ & Pastagem de Brachiaria decumbens, ração comercial \\
& Armazém (Surto 9) & $6 / 15$ & Farelo de trigo, milho e silagem de milho \\
Arroio Trinta (Surto 10) & $25 / 35$ & Pastagem de azevém \\
& Água Doce (Surto 11) & $10 / 10$ & Pastagem de azevém \\
& Lages (Surto 12) & $1 / 20$ & $5 / 10$
\end{tabular}

hiperemia e ou, rachaduras da pele da coroa do casco e da extremidade da cauda. Nos surtos ocorridos em Bom Retiro/SC, os quais ocorreram na mesma propriedade em dois anos consecutivos, as lesões eram muito graves, chegando a ocorrer perda das extremidades dos membros.

Os sinais clínicos e as lesões observadas na doença espontânea foram semelhantes aos descritos para intoxicação por C. purpurea, que desde a Idade Média (Mantle 1969) é conhecido por possuir ergotoxinas capazes de promover vasoconstrição periférica, tendo como consequência necrose de extremidades em baixas temperaturas ambiente e hipertermia nas estações quentes (Woods et al. 1966).

Dos três surtos da forma convulsiva, dois Surtos (11 e 13) os bovinos eram de raças de corte e um, (Surto 12) eram bovinos da raça Jersey. Os bovinos do Surto 13 eram alimentados com feno de aveia em fase de maturação das sementes e os dos Surtos 11 e 12 estavam em pastagens de azevém (Lolium sp.) na fase de maturação das sementes e em parte destas havia contaminação por um fungo morfologicamente semelhante ao $C$. purpurea. Os principais sinais clínicos foram taquipneia, tremores e espasmos musculares às vezes seguidos de convulsão, diarreia, morte ou recuperação após substituição da alimentação. Nos Surtos 11 e 12 dois bovinos foram necropsiados, porém não apresentaram alterações macroscópicas e microscópicas. Estes sinais também foram descritos por Burfening (1973) como ergotismo nervoso causado pela ingestão de C. purpurea.

\section{Intoxicação experimental}

A partir do isolamento realizado no Laboratório de Fitopatologia do Centro de Ciência Agroveterinárias, obteve-se através do exame microscópico a seguinte caracterização morfológica do fungo: os conídios apresentam coloração marrom claro e marrom avermelhado, cilíndricos a fusóides, lisos e levemente curvados, com célula basal contendo hilo inconspícuo, com 2 a 6 septos medindo 28-60 x 8,5-14,0 $\mathrm{mm}$. Por meio das características analisadas, os conídios são similares ao fungo Bipolaris australis (Alcorn 1982, Silvanesan 1987) detectado em inflorescências de espécies de Sporobolus e outras gramíneas. Esse fungo apresenta como sinônimo homotálico Curvularia australis (Alcorn 1982, Tan etal. 2014). A descrição dos sinais manifestados pelos bovinos na intoxicação experimental por inflorescências de $S$. indicus infectadas por Bipolaris sp. estão descritos no Quadro 3.

Experimentalmente a doença foi reproduzida com a ingestão de inflorescências de $S$. indicus infectadas por Bipolaris sp. em doses a partir de $0,1 \mathrm{~g} / \mathrm{kg}$. Desta forma, suspeita-se que este fungo é sim capaz de produzir hipertermia e vasoconstrição de extremidades levando a manifestações de sinais clínicos semelhantes aos produzidos por Claviceps purpurea. As alterações observadas no Bovino 1 foram hipertermia a partir do segundo dia de ingestão das inflorescências de $S$. indicus infectadas por Bipolaris sp. (Fig.1), mantendo-se assim até o sétimo dia, mesmo após o término do consumo das inflorescências contaminadas. Nesse período também houveram picos de taquicardia e taquipneia, chegando a $129 \mathrm{bpm}$ e $78 \mathrm{bpm}$ respectivamente. No sexto e no sétimo dia após o período da ingestão das inflorescências o bovino apresentou diarreia. 0 Bovino 2 teve o pico de temperatura no sexto dia de ingestão das inflorescências, porém no restante do período esse parâmetro não teve grande variação. Quanto à frequência cardíaca, as maiores alterações ocorreram no sexto e no sétimo dia, da mesma forma que a frequência respiratória. Estas alterações ocorreram nos dias em que a temperatura do ambiente teve maior elevação durante o período de ingestão. No sexto dia após o início do fornecimento das inflorescências o bovino apresentou diarreia.

No Bovino 3 que consumiu as inflorescências por um período maior, a temperatura corporal foi elevada a partir do quinto dia após a ingestão mantendo-se assim em vários dias, inclusive após o término da ingestão. As alterações nas frequências cardíaca e respiratórias iniciaram no dia 4, mantendo-se altas durante vários dias. Essas alterações coincidiram com o aumento da temperatura ambiente, conforme ilustrado na 
Quadro 3. Alterações clínicas em bovinos após a ingestão das inflorescências de Sporobolus indicus infectadas por Bipolaris sp.

\begin{tabular}{|c|c|c|c|c|c|}
\hline $\begin{array}{c}\text { Identificação do } \\
\text { bovino }\end{array}$ & Dose g/dia & Tempo de ingestão & Sinais clínicos & $\begin{array}{c}\text { Inicio dos sinais clínicos após } \\
\text { a ingestão }\end{array}$ & Lesões em extremidades \\
\hline Bovino 1 & 62 & 4 & $\begin{array}{l}\text { Hipertemia, taquicardia, } \\
\text { taquipneia, diarreia }\end{array}$ & $\begin{array}{l}2^{\circ} \text { dia: Hipertermia, } \\
\text { taquicardia, taquipneia } \\
6^{0} \text { dia: Diarreia }\end{array}$ & Sem alterações \\
\hline Bovino 2 & 45 & 7 & $\begin{array}{l}\text { Hipertemia, taquicardia, } \\
\text { taquipneia, diarreia }\end{array}$ & $\begin{array}{l}\text { 6o dia: Taquicardia, } \\
\text { taquipneia, diarreia }\end{array}$ & Sem alterações \\
\hline Bovino 4 & 35 & 30 & $\begin{array}{l}\text { Hipertemia, taquipneia, } \\
\text { diarreia }\end{array}$ & $\begin{array}{l}\text { 3 dia: Taquipneia } \\
\text { 3ㅇdia: Diarreia } \\
\text { 9o dia: Hipertermia }\end{array}$ & Sem alterações \\
\hline
\end{tabular}

Figura 1. 0 bovino apresentou lesões hiperêmicas na coroa dos cascos em três dos quatro membros, associado à perda dos pêlos da extremidade da cauda (Fig.2). Estas lesões sugerem vasoconstrição de extremidades como as descritas em intoxicações por C. purpurea (Belser-Ehrlich et al. 2013). 0 bovino também apresentou diarreia no terceiro e no quarto dia após o início do fornecimento das inflorescências.

As alterações manifestadas pelo Bovino 4 estão descritas na Figura 1, onde se observa que o aumento da temperatura corporal coincidiu com o aumento da temperatura ambiente. Houve ainda, alguns dias em que a frequência respiratória avaliada no período mais quente do dia esteve acima dos valores normais, porém a frequência cardíaca não teve alterações significativas. O bovino apresentou diarreia no terceiro e no vigésimo nono dia após o início do fornecimento das inflorescências. 0 Bovino 5 não teve alterações significativas ou fora dos parâmetros fisiológicos considerados por Stöber (1993), como normais para a espécie. É relevante ressaltar que no período de experimentação a temperatura ambiental não ultrapassou $22^{\circ} \mathrm{C}$, tendo valores médios entre 12 e $16^{\circ} \mathrm{C}$ (Epagri 1991).

Na reprodução experimental, o quadro clínico de hipertermia ocorreu nos períodos mais quentes do dia, e está de acordo com o descrito para a intoxicação por C. purpurea (Al-Tamimi et al. 2003). Já o Bovino 5 que ingeriu as inflorescências infectadas nos dias mais amenos não manifestou estes sinais. As lesões na cauda e na coroa do casco descritas no Bovino 3 foram leves. Isto pode ser explicado pelas temperaturas amenas registradas no período.

Diarreia foi um sinal clínico observado em quatro dos cinco bovinos. Este achado está de acordo com os sinais clínicos descritos Bourke et al. (2000) na intoxicação por C. purpurea.

Baseado nos achados clínicos de hipertermia, taquicardia, taquipneia e hiperemia do rodete coronário, sugere-se que o fungo B. australis, isolado de todas as amostras de $S$. indicus, possua uma ou mais substâncias do grupo das ergotoxinas capazes de promover vasoconstrição periférica. No presente estudo não foi isolado $C$. purpurea das inflorescências de $S$. indicus. 0 isolamento de $B$. australis sugere que este produza micotoxinas com ações semelhantes a C. purpurea.

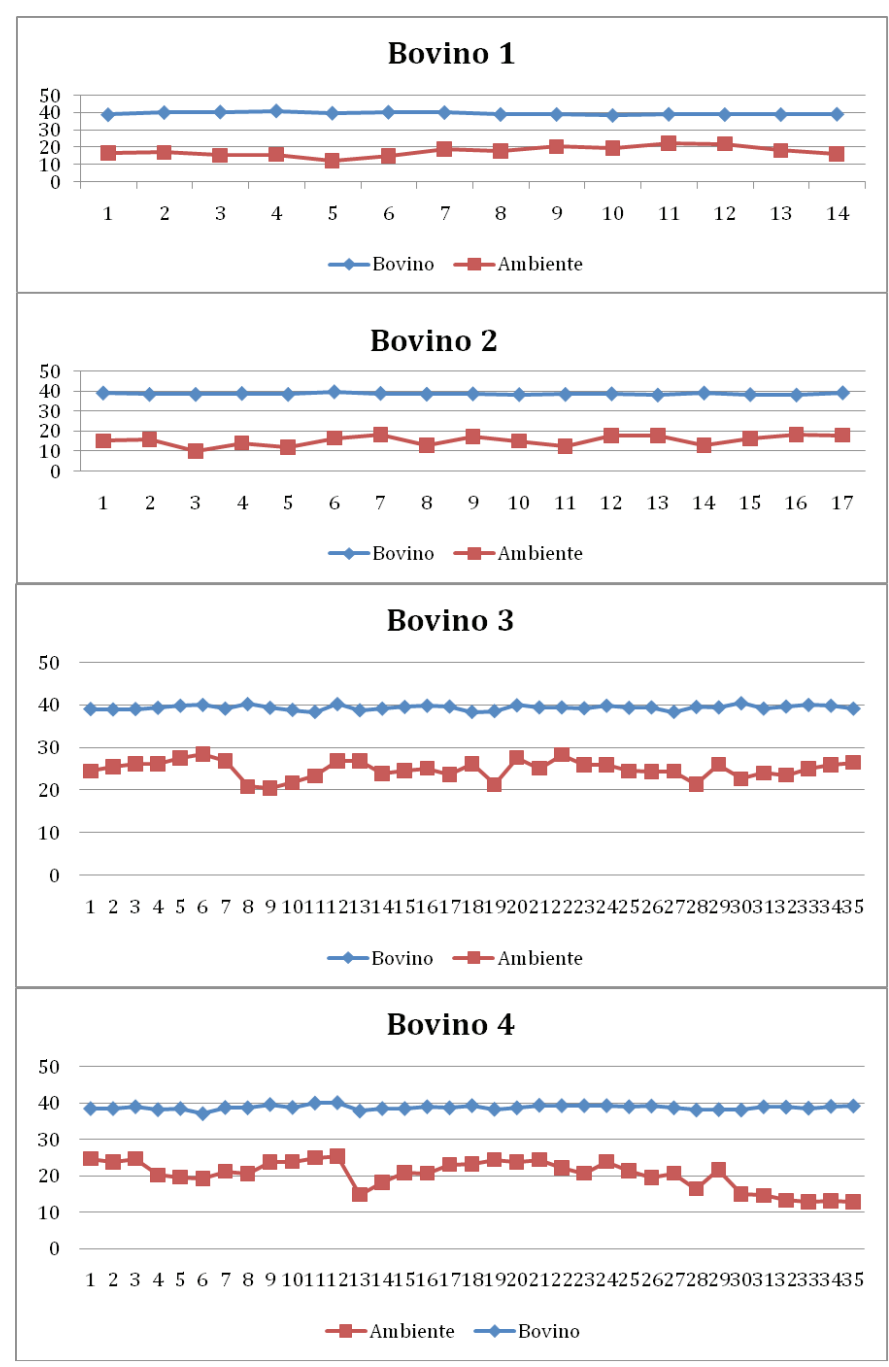

Fig.1. Variação da temperatura do Bovino 1, 2, 3 e 4 submetido a intoxicação experimental por inflorescências de Sporobolus indicus infectadas por Bipolaris sp. em função da temperatura do ambiente. 

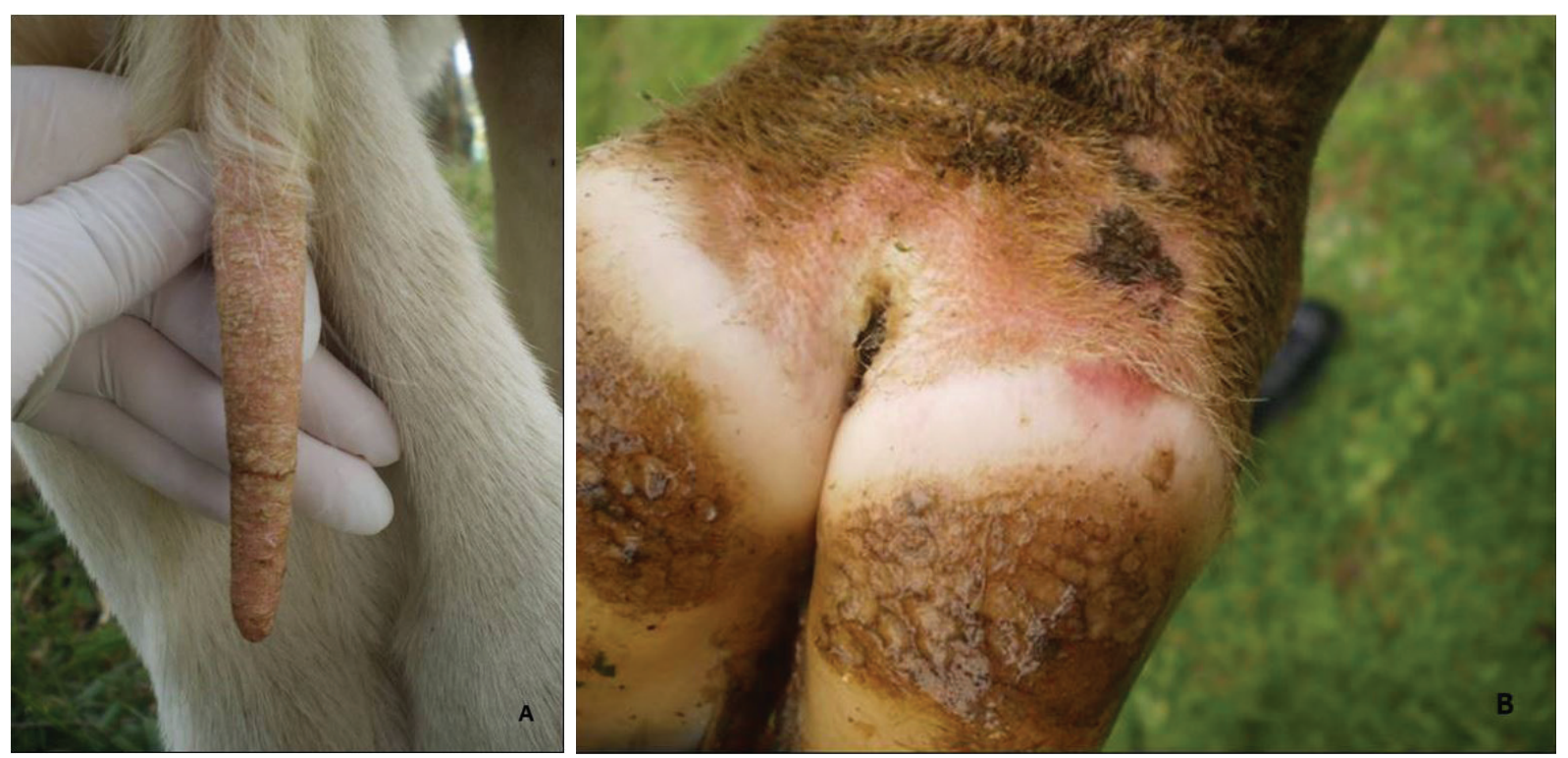

Fig.2. (A) Alopecia da extremidade da cauda do Bovino 3 submetido a intoxicação experimental por inflorescências de Sporobolus indicus infectadas por Bipolaris sp. Fissuras e crostas na extremidade distal. (B) Hiperemia na coroa do casco.

Em bovinos a hipertermia, taquicardia, taquipneia, hiperemia da coroa do casco e perda de pêlos da extremidade da cauda, causadas pela ingestão de sementes de $S$. indicus contaminadas por B. australis deve ser diferenciado de outras enfermidades. Dentre estas, podem ser citadas a intoxicação por C. purpurea (Belser-Ehrlich et al. 2013), cujos sinais clínicos e lesões são semelhantes; Neotyphodium coenophialum, um fungo endofítico que infecta festuca (Festuca arundinacea) onde causa hipertermia, baixo desenvolvimento, diminuição da produção de leite e do desempenho reprodutivo (Bhusari et al. 2007). Deve-se diferenciar também da intoxicação pelo cogumelo Ramaria flavo-brunnescens que pode causar entre outros sinais o desprendimento dos pêlos da extremidade da cauda, salivação, lesões necróticas da coroa do casco e perda de peso (Barros et al. 2006).

Os sinais clínicos e as lesões observadas na doença espontânea foram semelhantes aos descritos para intoxicação por C. purpurea, que desde a Idade Média (Mantle 1969) é conhecido por possuir ergotoxinas capazes de promover vasoconstrição periférica, tendo como consequência necrose de extremidades em baixas temperaturas ambiente e hipertermia nas estações quentes (Woods et al. 1966). De acordo com a literatura, esse fungo possui ergotoxinas que agem no endotélio dos capilares sanguíneos promovendo a vasoconstrição periférica, tendo como consequências gangrena de extremidades, hipertermia e transtornos reprodutivos (Burfening 1973, Appleyard 1986, Riet-Correa et al. 1988).

\section{CONCLUSÕES}

Em Santa Catarina o uso de aveia (Avena sativa e A. strigosa), azevém (Lolium sp.), no sistema de pastoreio direto e, ou fornecimento destas sementes no cocho é altamente difundido e é responsável por produzir surtos de ergotismo em bovinos.

0 fungo Bipolaris sp. é contaminante de inflorescências de Sporobolus indicus e pode ser tóxico para bovinos. Em doses superiores $0,1 \mathrm{~g} / \mathrm{kg}$ do capim infectado produziu hipertermia, taquicardia, taquipneia, hiperemia da coroa do casco e perda de pêlos da extremidade da cauda.

0 diagnóstico da intoxicação por Bipolaris sp. é feito através da observação do quadro clínico, das lesões e dos dados epidemiológicos associados à presença de inflorescências de $S$. indicus infectadas pelo fungo.

Agradecimentos.- Ao Programa de Bolsas de Estudo de Educação Superior Uniedu/Pós-graduação- Fundo de Apoio à Manutenção e ao Desenvolvimento da Educação Superior (FUMDES) pela concessão da bolsa de mestrado.

\section{REFERÊNCIAS}

Alcorn J.L. 1982. Ovariicolous Bipolaris species on Sporobolus and other grasses. Mycotaxon 15:20-48.

Alexopoulos C.J. \& Mims C.W. 1996. Introductory Mycology. 4th ed. John Wiley, New York, p.338-343.

Al-Tamimi H.J., Rottinghaus G.E., Spiers D.E., Spain J., Chatman D., Eichen P.A. \& Carson T.L. 2003. Thermoregulatory response of dairy cows fed ergotized barley during summer heat stress. J. Vet. Diagn. Invest. 15(4):355-360. http://dx.doi.org/10.1177/104063870301500409. PMid:12918817.

Appleyard W.T. 1986. Outbreak of bovine abortion attributed to ergot poisoning. Vet. Rec. 118(2):48-49. http://dx.doi.org/10.1136/vr.118.2.48. PMid:3946070.

Barros R.R., Irigoyen L.F., Kommers G.D., Rech R.R., Fighera R.A. \& Barros C.S.L. 2006. Intoxicação por Ramaria flavo-brunnescens (Clavariaceae) em bovinos. Pesq. Vet. Bras. 26(2):87-96. http://dx.doi.org/10.1590/ S0100-736X2006000200005.

Belser-Ehrlich S., Harper A., Hussey J. \& Hallock R. 2013. Human and cattle ergotism since 1900: symptoms, outbreaks, and regulations. Toxicol. Ind. Health 29(4):307-316. http://dx.doi.org/10.1177/0748233711432570. PMid:22903169.

Bhusari S., Liu Z., Hearne L.B., Spiers D.E., Lamberson W.R. \& Antoniou E. 2007. Expression profiling of heat stress effects on mice fed ergot 
alkaloids. Toxicol. Sci. 95(1):89-97. http://dx.doi.org/10.1093/toxsci/ kfl142. PMid:17093207.

Bourke C.A. 2003. Evidence that enforced sunlight exposure can cause hyperthermia in cattle ingesting low levels of ergot of rye (Claviceps purpurea), when air temperature and humidity conditions are only moderate. Aust. Vet. J. 81(9):553-558. http://dx.doi.org/10.1111/j.1751-0813.2003. tb12886.x. PMid:15086095.

Bourke C.A., Bailey G.D. \& Kemp J.B. 2000. The case for solar light radiation being more significant than ambient temperature in producing lethal hyperthermic ergotism in cattle. Aust. Vet. J. 78(9):618-621. http://dx.doi. org/10.1111/j.1751-0813.2000.tb11936.x. PMid:11022289.

Burfening P.J. 1973. Ergotism. J. Am. Vet. Med. Assoc. 163(11):1288-1290. PMid:4761655.

Copetti M.V., Santurio J.M., Boeck A.A.P., Silva R.B., Bergermaier L.A., Lubeck I., Leal A.B.M., Leal A.T., Alves S.H. \& Ferreiro L. 2002. Agalactia in mares fed with grain contaminated with Claviceps purpurea. Mycopathologia 154(4):199200. http://dx.doi.org/10.1023/A:1016379302055. PMid:12206321.

Coppock R.W., Mostrom M.S., Simon J., Mckenna D.J., Jacobsen B. \& Szlachta H. 1989. Cutaneous ergotism in a herd of dairy calves. J. Am. Vet. Med. Assoc. 194(4):549-551. PMid:2921208.

El Khizzi N., Bakheshwai S. \& Parvez S.. 2010. Bipolaris: a plant pathogen causing human infections, an emerging problem in Saudi Arabia. Res. J. Microbiol. 5(3):212-217. http://dx.doi.org/10.3923/jm.2010.212.217.

Epagri 1991. Centro de Informações de Recursos Ambientais e de Hidrometereologia de Santa Catarina. Disponível em <http://ciram.epagri. sc.gov.br/index.php?option=com_conten\& view=article\&id=98\&Itemid=198> Acesso em 15 maio 2015.

Fontes A.S. \& Abreu A.C.S. 2015. Ethmoid-orbital mycetoma caused by Bipolaris sp. Acta Otorrinolaringologica Española 66(2):e1-e3. http:// dx.doi.org/10.1016/j.otorri.2013.10.002. PMid:24418151.

Giri D.K., Sims W.P., Sura R., Cooper J.J., Gavrilov B.K. \& Mansell J. 2011. Cerebral and renal phaeohyphomycosis in a dog infected with Bipolaris species. Vet. Pathol. 48(3):754-757. http://dx.doi.org/10.1177/0300985810375239. PMid:20574070.

Gourley D.S., Whisman B.A., Jorgensen N.L., Martin M.E. \& Reid M.J. 1990. Allergic Bipolaris sinusitis: clinical and immunopathologic characteristics. J. Allergy Clin. Immunol. 85(3):583-591. http://dx.doi.org/10.1016/00916749(90)90097-N. PMid:2312993.

Ilha M.R.S., Riet-Correa F. \& Barros C.S.L. 2001. Síndrome distérmica (hipertermia) em bovinos associada à intoxicação por Claviceps purpurea. Pesq. Vet. Bras. 21(2):81-86. http://dx.doi.org/10.1590/S0100-736X2001000200007.

Ilha M.R.S., Loretti A.P. \& Barros C.S.L. 2003. Hyperthermic syndrome in dairy cattle associated with consumption of Ergots of Claviceps purpurea in Southern Brazil. Vet. Hum. Toxicol. 45(3):140-145. PMid:12776790.

Jessep T.M., Dent C.H.R., Kemp J.B., Christie B., Ahrens P.J., Burgess L.W. \& Bryden W.L. 1987. Bovine idiopathic hyperthermia. Aust. Vet. J. 64(11):353-354. http://dx.doi.org/10.1111/j.1751-0813.1987.tb06068.x. PMid:3447580.

Khalloub P., Diab S., Licoff N., Bengolea A., Lázaro L., Cantón G. \& Odriozola E. 2007. Efecto del consumo de Claviceps purpurea em bovinos em engorde. Sitio Argentino de Produção Animal 88(2):68-72.

Kissmann K.G. \& Groth D. 1997. Plantas Infectantes e Nocivas. $2^{\underline{a}}$ ed. BASF, São Paulo, p.766-769.

Koshy S. \& Daniel E. 2002. Bipolaris keratomycosis in a leprosy patient: a case report. Leprosy Review 73(1):76-78. PMid:11969130.

Lorenzi H. 2008. Plantas Daninhas do Brasil: Terrestres, Aquáticas, Parasitas e Tóxicas. $4^{\underline{a}}$ ed. Instituto Plantarum de Estudos da Flora, Nova Odessa, p.552-553.
Luzzardi G.C. \& Oliveira B.S. 1973. The occurrence of ergot in several forrage grasses in Southern Brazil. Anais 8o Congresso Anual da Sociedade Brasileira de Fitopatologia, Pelotas, RS, p.13. (Resumo)

Mantle P.G. \& Gunner D.E. 1965a. Abortion associated with ergotised pastures. Vet. Rec. 77:885-886. PMid:14331490.

Mantle P.G. \& Gunner D.E. 1965b. Abortion associated with ergotised pastures. Vet. Rec. 77:911-912. PMid:14331490.

Mantle P.G. 1969. The role of alkaloids in the poisoning of mammals by sclerotia of Claviceps spp. J. Stored Prod. Res. 5(3):237-244. http://dx.doi. org/10.1016/0022-474X(69)90038-1.

McGinnis M.R., Rinaldi M.G. \& Winn R.E. 1986. Emerging agents of phaeohyphomycosis: pathogenic species of Bipolaris and Exserohilum. J. Clin. Microbiol. 24(2):250-259. PMid:3745423.

McKeon F.W. \& Egan D.A. 1971. Lameness in cattle fed ergotised silage. Irish Vet. J. 25:67-69.

Peet R.L., McCarthy M.R. \& Barbetti M.J. 1991. Hyperthermia and death in feedlot cattle associated with the ingestion of Claviceps purpurea. Aust. Vet. J. 68(3):121. http://dx.doi.org/10.1111/j.1751-0813.1991.tb00777.x. PMid:2043090.

Quinn M.V.B., Maerkey B.K., Carter M.E., Donnelly W.J.C., Leonard F.C. \& Maguire D. 2005a. Infecções oportunistas causadas predominantemente por fungos feóides, p.257-259. In: Ibid. (Eds), Microbiologia Veterinária e Doenças Infecciosas. Artmed, Porto Alegre.

Quinn M.V.B., Maerkey B.K., Carter M.E., Donnelly W.J.C., Leonard F.C. \& Maguire D. 2005b. Micotoxinas e micotoxicoses, p.260-268. In: Ibid. (Eds), Microbiologia Veterinária e Doenças Infecciosas. Artmed, Porto Alegre.

Radostits O.M., Gay C.C., Blood D.C. \& Hinchcliff K.W. 2002. Doenças causadas por toxinas de plantas, fungos cianofitas, clavibactérias e por venenos de carrapatos e animais vertebrados, p.1472-1547. In: Radostits O.M., Gay C.C., Blood D.C. \& Hinchcliff K.W. (Eds), Clínica Veterinária: um tratado de doenças de bovinos, ovinos, caprinos, suínos e equídeos. $9^{a}$ ed. Guanabara Koogan, Rio de Janeiro.

Rao A., Forgan-Smith F., Miller S. \& Haswell H. 1989. Phaeohyphomycosis of the nasal sinuses caused by Bipolaris species. Pathology 21(4):280-281. http://dx.doi.org/10.3109/00313028909061075. PMid:2633117.

Riet-Correa F. 1993. Micotoxicoses que produzem ergotismo, p.227-239. In: Riet-Correa F., Mendez M.C. \& Schild A.L. (Eds), Intoxicações por Plantas e Micotoxinas em Animais Domésticos. Editorial Hemisfério Sul do Brasil, Pelotas.

Riet-Correa F., Mendez M.C., Schild A.L., Bergamo P.N. \& Flores W.N. 1988. Agalactia, reproductive problems and neonatal mortality in horses associated with the ingestion of Claviceps purpurea. Aust. Vet. J. 65(6):192-193. http://dx.doi.org/10.1111/j.1751-0813.1988.tb14301.x. PMid:3415622.

Rissi D.R., Rech R.R., Pierezan F., Gabriel A.I., Trost M.E., Brum J.S., Kommers G.D. \& Barros C.S.L. 2007. Intoxicações por plantas e micotoxinas associadas a plantas em bovinos no Rio Grande do Sul: 461 casos. Pesq. Vet. Bras. 27(7):261-268. http://dx.doi.org/10.1590/S0100-736X2007000700002.

Ross A.D., Bryden W.L., Bakau W. \& Burgess L.W. 1989. Induction of heat stress in beef cattle by feeding the ergots of Claviceps purpurea. Aust. Vet. J. 66(8):247-249. http://dx.doi.org/10.1111/j.1751-0813.1989.tb13581.x. PMid:2590138.

Saha R. \& Das S. 2005. Bipolaris keratomycosis. Mycoses 48(6):453-455. http://dx.doi.org/10.1111/j.1439-0507.2005.01151.x. PMid:16262886.

Saubolle M.A. \& Sutton J. 1996. The Dematiaceous Fungal genus Bipolaris and its role in human disease. Clin. Microbiol. Newsletter 18(1):1-6. http:// dx.doi.org/10.1016/S0196-4399(96)80019-5.

Schneider D.J., Miles C.O., Garthwaite I., Van Halderen A., Wessels J.C. \& Lategan H.J. 1996. First report of field outbreaks of ergot-alkaloid toxicity in South Africa. Onderstepoort J. Vet. Res. 63(2):97-108. PMid:8966013. 
Silvanesan A. 1987. Graminicolous species of Bipolaris, Curvularia, Drechslera, Exserohilum and their teleomorphs. Mycological Papers, CAB International Mycological Institute, Wallingford, 158:261.

Sousa M.E., Araújo M.A.S., Mota R.A., Porto W.J.N., Souza A.K.P., Santos J.L. \& Silva P.P. 2011. Microbiota fúngica da conjuntiva ocular de equinos clinicamente saudáveis pertencentes à Cavalaria da Polícia Militar de Alagoas. Braz. J. Microbiol. 42(3):1151-1155. http://dx.doi.org/10.1590/ S1517-83822011000300038. PMid:24031735.

Stöber M. 1993. Identificação, anamnese, regras básicas da técnica de exame clínico geral, p.44-80. In: Dirksen G., Grunert H.-D. \& Stöber M. (Eds), Exame Clínico dos Bovinos. 3a ed. Guanabara Koogan, Rio de Janeiro.

Tan Y.P., Madrid H., Crous P.W. \& Shivas R.G. 2014. Johnalcornia gen. et comb. nov., and new combinations and synonymies in Bipolaris and Curvularia. Australasian Plant Pathology 43(6):589-603. http://dx.doi.org/10.1007/ s13313-014-0315-6.
Teran C.G., Downes K. \& Medows M. 2014. Fatal Bipolaris spicifera Infection in an immunosuppressed child. BMJ Case Rep. 2014:bcr2013009703. http:// dx.doi.org/10.1136/bcr-2013-009703. PMid:24493105.

Vital M.A.B.F. \& Acco A. 2006. Agonistas e antagonistas adrenérgicos, p.81-96. In: Spinosa H.S., Gorniak S.L. \& Bernardi M.M. (Eds), Farmacologia Aplicada à Medicina Veterinária. 4a ed. Guanabara Koogan, Rio de Janeiro.

Waurzyniak B.J., Hoover J.P., Clinkenbeard K.D. \& Welsh R.D. 1992. Dual systemic mycosis caused by Bipolaris spicifera and Torulopsis glabrata in a dog. Vet Pathol. 29(6):566-569. http://dx.doi.org/10.1177/030098589202900620. PMid:1448909.

Woods A.J., Jones J.B. \& Mantle P.G. 1966. An outbreak of gangerous ergotism in cattle. Vet. Rec. 78(22):742-749. http://dx.doi.org/10.1136/vr.78.22.742. PMid:6006906. 